\title{
Adiponectin ameliorates the apoptotic effects of paraquat on alveolar type II cells via improvements in mitochondrial function
}

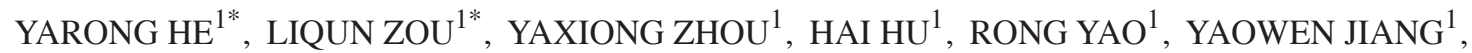 \\ WAYNE BOND LAU ${ }^{2}$, TUN YUAN ${ }^{3}$, WEN HUANG $^{4}, \mathrm{ZHI} \mathrm{ZENG}^{1 * *}$ and YU CAO ${ }^{1 * *}$ \\ ${ }^{1}$ Emergency Medicine Department, West China Hospital, Sichuan University, Chengdu, Sichuan 610041, P.R. China; \\ ${ }^{2}$ Emergency Medicine Department, Thomas Jefferson University Hospital, Philadelphia, PA 19107, USA; \\ ${ }^{3}$ National Engineering Research Center for Biomaterials, Sichuan University, Chengdu, Sichuan 610064; \\ ${ }^{4}$ Laboratory of Ethnopharmacology, Institute for Nanobiomedical Technology and Membrane Biology, \\ Regenerative Medicine Research Center, West China Hospital, Sichuan University, Chengdu, Sichuan 610041, P.R. China
}

Received June 11, 2015; Accepted April 19, 2016

DOI: $10.3892 / \mathrm{mmr} .2016 .5328$

\begin{abstract}
Previous studies have demonstrated that excessive reactive oxygen/nitrogen species (ROS/RNS)-induced apoptosis is an important feature of the injury to the lung epithelium in paraquat (PQ) poisoning. However the precise mechanisms of PQ-induced dysfunction of the mitochondria, where ROS/RNS are predominantly produced, remain to be fully elucidated. Whether globular adiponectin (gAd), a potent molecule protective to mitochondria, regulates the mitochondrial function of alveolar type II cells to reduce PQ-induced ROS/RNS production remains to be investigated. The current study aimed to investigate the precise mechanisms of $\mathrm{PQ}$ poisoning in the mitochondria of alveolar type II cells, and to elucidate the role of $\mathrm{gAd}$ in protecting against $\mathrm{PQ}$-induced lung epithelium injury. Therefore, lung epithelial injury was induced by PQ co-culture of alveolar type II A549 cells for $24 \mathrm{~h}$. gAd was administrated to and removed from the injured cells in after $24 \mathrm{~h}$. PQ was observed to reduce cell viability and increase apoptosis by $\sim 1.5$ fold in A549 cells. The oxidative/nitrative stress, resulting from ROS/RNS and disordered mitochondrial function were evidenced by increased $\mathrm{O}_{2}^{-}$, NO production and reduced mitochondrial membrane
\end{abstract}

Correspondence to: Dr Zhi Zeng or Professor Yu Cao, Emergency Medicine Department, West China Hospital, Sichuan University, 37 Guoxue Road, Chengdu, Sichuan 610041, P.R. China

E-mail: 530291451@qq.com

E-mail: yuyuer@126.com

${ }^{* * * *}$ Contributed equally

Abbreviation: ROS/RNS, reactive oxygen/nitrogen species; PQ, paraquat; gAd, globular adiponectin; ATP, adenosine 5'-triphosphate; AMPK, adenosine 5'-monophosphate-activated protein kinase

Key words: paraquat, gAd, reactive oxygen/nitrogen species, apoptosis, AMPK potential $(\Delta \Psi)$, adenosine 5 '-triphosphate (ATP) content in PQ-poisoned A549 cells. gAd treatment significantly reversed the PQ-induced cell injury and mitochondrial dysfunction in A549 cells. The protective effects of gAd were partly abrogated by an adenosine 5'-monophosphate-activated protein kinase (AMPK) inhibitor, compound $\mathrm{C}$. The results suggest that reduced $\Delta \Psi$ and ATP content may result in PQ-induced mitochondrial dysfunction of the lung epithelium, which constitutes a novel mechanism for gAd exerting pulmonary protection against $\mathrm{PQ}$ poisoning via AMPK activation.

\section{Introduction}

Previous studies have demonstrated that apoptosis is an important process in injured to the lung epithelium in paraquat $(\mathrm{PQ})$ poisoning $(1,2)$. The mechanisms of apoptosis are highly complex and involve oxidative/nitrative stress impairment to the function of mitochondria with the reduction of the mitochondrial membrane potential $(\Delta \Psi)$, resulting in an increase of superoxide formation and reduced production of adenosine 5'-triphosphate (ATP) $(3,4)$. Previous studies suggest that adenosine 5'-monophosphate-activated protein kinase (AMPK) is one of the most important molecules in the stimulation of ATP production and protection against oxidative/nitrative stress in mitochondria $(5,6)$. However, whether dysfunction of the mitochondria and reduction of ATP via inactivation of AMPK serve crucial roles in the PQ-induced apoptosis of alveolar epithelial type II cells remains to be fully elucidated.

Adiponectin (APN), an adipocytokine predominantly secreted by adipocytes and found at high levels in the plasma, has been reported to exhibit anti-insulin resistance and anti-inflammatory and anti-apoptotic properties (7-9). Circulating APN is present as trimeric, hexameric or oligomeric complexes of monomers, and cleavage to produce the C-terminal globular domain, gAd, has been proposed as an important regulatory step in the action of APN. This is due to the fact that this C-terminal fragment has been reported to mediate potent physiological effects $(10,11)$. Previous studies have suggested that the potential role of gAd against apoptosis 
is mediated via the activation of AMPK (12-14). APN-knockout mice exhibit inhibition of AMPK, a reduction in the levels of ATP, an increase of mitochondrial swelling and ROS/RNS production, resulting in augmented apoptosis, while administration of gAd reversed these effects. The protective effects of gAd have been reported to be reversed by administration of the AMPK inhibitor (compound C) (15). It remains unclear whether APN may protect alveolar type II cells from PQ poisoning via activation of AMPK and increasing the production of ATP in mitochondria.

Therefore, the aims of the current study were: i) To determine whether mitochondrial function is impaired and ATP is reduced in PQ-poisoned alveolar type II cells via inactivation of AMPK; ii) to determine the effect of gAd against PQ-induced apoptosis and in alveolar type II cells; and if so, iii) whether gAd mediates protective effects via activation of AMPK and increasing production of ATP.

\section{Materials and methods}

Materials. All reagents were purchased from Sigma-Aldrich (St. Louis, MO, USA), unless otherwise specified. RPMI 1640 medium was obtained from Gibco; Thermo Fisher Scientific, Inc. (Waltham, MA, USA). Penicillin/streptomycin was obtained from Wisent, Inc. (Saint-Bruno, QC, Canada). PQ was purchased from Tokyo Chemical Industry Co., Ltd. (Tokyo, Japan). The globular domain of APN (gAd) was purchased from Beijing Adipobiotech, Inc. (Beijing, China). Compound C, an AMPK Inhibitor, was obtained from Merck Millipore (Darmstadt, Germany). The Annexin V-Fluorescein Isothiocyanate (FITC) Apoptosis Detection kit I was from Nanjing KeyGen Biotech Co., Ltd. (Nanjing, China). JC-1 and dihydroethidium (DHE) were purchased from Molecular Probes; Thermo Fisher Scientific, Inc. The Nitrate/Nitrite Colorimetric Assay kit was from Nanjing Jiancheng Biochemical Reagent Co. (Nanjing, China). The ATP Bioluminescent Somatic Cell assay kit was from Sigma-Aldrich.

Cell cultures and treatments. The human lung adenocarcinoma cell line A549 (Shanghai Institute of Biochemistry and Cell Biology, Shanghai, China) was grown in a monolayer culture in RPMI 1640 medium supplemented with $10 \%$ fetal bovine serum, $1 \%$ glutamine, $1 \%$ (v/v) streptomycin/penicillin. The culture medium was refreshed two to three times per week and was subcultured upon reaching $80 \%$ confluence. The cells were then seeded onto flat-bottom six-well or 96-well plates with growth medium at $37^{\circ} \mathrm{C}$ in a humidified atmosphere at $5 \%$ $\mathrm{CO}_{2}$, followed by administration of PQ at $300 \mu \mathrm{M}$ for $24 \mathrm{~h}$ for the PQ, PA and PCA groups. Subsequently, gAd $(2.5 \mu \mathrm{g} / \mathrm{ml})$ was added and the cells were cultured for another $24 \mathrm{~h}$, in order to investigate the role of gAd in PQ-induced cytotoxicity. PCA group cells were also treated with compound C $(1 \mu \mathrm{M})$ for 30 min prior to the addition of $\mathrm{PQ}$. The optimal doses and time points for $\mathrm{gAd}, \mathrm{PQ}$ and compound $\mathrm{C}$ were determined through preliminary experiments (data not shown).

Measurement of cell viability. The 3-(4, 5-methylthiazol-2-yl)-2,5-diphenyltetrazolium bromide (MTT) assay was conducted as previously described (16). Briefly, following PQ challenge, culture media was refreshed with media containing MTT reagent $(5 \mathrm{mg} / \mathrm{ml})$ and cells were incubated under standard conditions for an additional $4 \mathrm{~h}$. The culture media was then carefully aspirated and $100 \mu \mathrm{l}$ dimethylsulfoxide was added per well to solubilize the formazan crystals. Following agitation, absorbance was measured spectrophotometrically at a wavelength of $490 \mathrm{~nm}$ using a Benchmark Plus Microplate Spectrophotometer (Bio-Rad Laboratories, Inc., Hercules, CA, USA). Viabilities of the challenged cells were expressed relative to control cells.

Measurement of necrosis and apoptosis. Apoptotic and necrotic cell death were measured using flow cytometry with the Annexin V-FITC Apoptosis Detection kit I (17). At $24 \mathrm{~h}$ after administration of gAd, cells were trypsinized (Thermo Fisher Scientific, Inc.) without ethylenediaminetetraacetic acid and labelled with the fluorochromes in the absence of light for $10 \mathrm{~min}$ at room temperature. Fluorescence was measured by flow cytometric analysis using a FACSCalibur flow cytometer (BD Biosciences, San Jose, CA, USA) to monitor green fluorescence (525 $\mathrm{nm}$ band-pass filter) for Annexin V-fluorescein conjugate and red fluorescence (575 $\mathrm{nm}$ band-pass filter) for PI respectively. The Annexin V-FITC-/PI-cell population was regarded as normal, while Annexin V-FITC+/PI-cells were taken as an indicator of early apoptosis, Annexin V-FITC+/PI+ as late apoptosis and Annexin V-FITC-/PI+ as necrosis. The data were analyzed using CellQuest software (BD Biosciences). A minimum of 30,000 gated events were acquired per sample.

Measurement of superoxide production. Production of superoxides was evaluated intracellularly using the superoxide-sensitive dye DHE (18). DHE is oxidized by superoxides to a novel product, which binds to DNA enhancing intracellular fluorescence. Culture medium was aspirated and cells were incubated with DHE (5 $\mu \mathrm{M}$ final concentration) for $10 \mathrm{~min}$ at $37^{\circ} \mathrm{C}$ in the dark. Following two rinses in phosphate-buffered saline, cells were photographed using an Axiostart 50 (Zeiss, Oberkochen, Germany) microscope equipped with a Canon PowerShot G5 epifluorescence attachment (Canon, Inc., Tokyo, Japan). Between five and six photographs were captured from each well. The fluorescence intensity values for each photo were determined using Adobe Photoshop software (version 4; Adobe Systems, Inc., San Jose, CA, USA).

Measurement of nitrites. Nitrites were measured in the culture supernatants by a colorimetric assay using a procedure based on the Greiss reaction with sodium nitrite as the standard (19). Culture medium was collected and stored at $-80^{\circ} \mathrm{C}$ until analysis with the Nitrate/Nitrite Colorimetric Assay kit, according to the manufacturer's instructions.

Measurement of $\Delta \Psi$. The membrane potential assay was conducted as previously described (20). The relative $\Delta \Psi$ was assessed using a laser scanning confocal microscope (SP8; Leica Microsystems GmbH, Wetzlar, Germany) analysis of cells stained with JC-1. Cells were incubated with $10 \mu \mathrm{g} / \mathrm{ml}$ $\mathrm{JC}-1$ for $30 \mathrm{~min}$ at $37^{\circ} \mathrm{C}$. Subsequent to applying the dye, the cells were scanned with the confocal microscope using a 10x objective lens. Fluorescence was excited by the 488-nm 


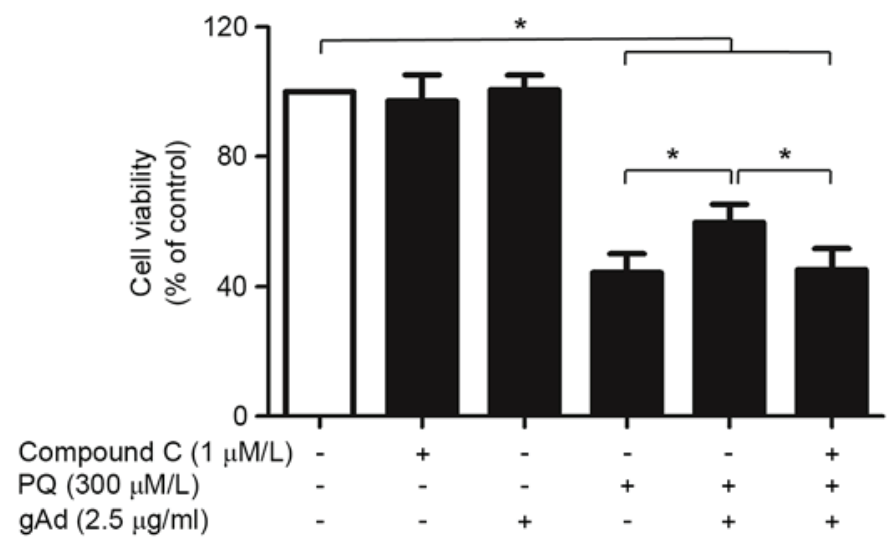

Figure 1. Cellular-protective effect of gAd on PQ-induced A549 cells. Cells were treated with $300 \mu \mathrm{M}$ PQ followed by $2.5 \mu \mathrm{g} / \mathrm{ml} \mathrm{gAd}$ with or without adenosine 5'-monophosphate-activated protein kinase inhibitor (compound C) pretreatment. The 3-(4,5-dimethylthiazol-2-yl)-2,5-diphenyltetrazolium bromide assay was performed at $24 \mathrm{~h}$ subsequent to the administration of gAd. The data are presented as the mean \pm standard deviation of five independent experiments, ${ }^{*} \mathrm{P}<0.05$. gAd, globular adiponectin; $\mathrm{PQ}$, paraquat.

line of an argon laser and the 543-nm line of a helium/neon laser. The red emission of the dye is due to a potential-dependent aggregation in the mitochondria, reflecting high $\Delta \Psi$. Green fluorescence indicates the monomeric form of JC-1, appearing in the cytosol following mitochondrial membrane depolarization, indicating low $\Delta \Psi$. The ratio of red/green indicated the mitochondrial membrane depolarization states of different cells.

Measurement of ATP. The amount of ATP was measured in the cultured cells using a luminometer (Bio Orbit 1251 Luminometer; Bio-Orbit, Turku, Finland) as previously described (21). Subsequent to exposure to different experimental conditions, cells were collected to react with different working solutions of from the ATP Bioluminescent Somatic Cell assay kit, according to the manufacturer's instructions. All reactions for a given sample were monitored simultaneously and calibrated with the addition of an ATP standard from the kit.

Statistics. Data are presented as the mean \pm standard error $(n=5)$ and were analyzed for statistical significance by one- or two-way analysis of variance with were least significant difference or Student-Newman-Keuls post hoc analyses. Statistical analysis and the significance of the data were determined using SPSS software, version 12.0 (SPSS, Inc., Chicago, IL, USA). P<0.05 was considered to indicate a statistically significant difference.

\section{Results}

$g$ Ad reduced $P Q$-mediated cytotoxicity. As presented in Fig. 1, PQ significantly reduced the cell viability of A549 cells, while gAd alleviated the PQ-induced cytotoxicity without any effects on the cells alone. However, pretreatment with compound $\mathrm{C}$ significantly reversed the protective effects of gAd on the viability reduced by $\mathrm{PQ}$.

gAd reduced $P Q$-mediated apoptosis. The results indicated that there was a low level of cell death in the control group (Fig. 2A). However, PQ was observed to induce apoptosis in $\sim 37.5 \%$ cells (Fig. 2B). However, gAd significantly reversed the PQ-induced cytotoxicity, reducing the percentage of apoptotic cells to $22.3 \%$ ( $\mathrm{P}<0.01)$. However, the pretreatment with compound $\mathrm{C}$ significantly reversed gAd's protective effect (Fig. 2).

gAd reduced $P Q$-mediated superoxide production. As presented in Fig. 3, control cultures generally exhibited low fluorescence intensity while PQ-treated cultures exhibited bright red fluorescence. This indicated that PQ significanlty increased $\mathrm{O}_{2}^{-}$production in cells compared with that of the controls $(\mathrm{P}<0.001)$. gAd inhibited the $\mathrm{O}_{2}^{-}$. production induced by PQ significanlty. However, pre-treatment with compound $\mathrm{C}$ partly reversed gAd's effects on $\mathrm{O}_{2}^{-\cdot}$ production.

$g$ Ad reduced $P Q$-mediated nitrite production. Addition of PQ to the cultures significantly increased nitrite production compared with the controls $(\mathrm{P}<0.01)$. Administration of $\mathrm{gAd}$ or compound $\mathrm{C}$ significantly reversed the increase, however pre-treatment with compound $\mathrm{C}$ did not reverse the effects of gAd on nitrite production (Fig. 4).

gAd reduced $P Q$-mediated $\Delta \Psi$ depolarization. Incubation with PQ depolarized the $\Delta \Psi$ significantly, resulting in a low ratio of red/green. Treatment with gAd repolarized the $\Delta \Psi$ of the cells, resulting in a greater ratio of red/green, compared with $\mathrm{PQ}$ group $(\mathrm{P}<0.01)$. However, compound $\mathrm{C}$ reversed the repolarization of $\mathrm{gAd}$, resulting in a lower ratio of red/green as compared with the gAd group $(\mathrm{P}<0.01 ;$ Fig. 5).

gAd partly reversed the $P Q$-mediated reduction in ATP. Addition of PQ significantly reduced the intracellular ATP concentration, as compared with the initial control levels: $21 \%$ after $24 \mathrm{~h}$, irrespective of the presence of other medium supplements or stimulation $(\mathrm{P}<0.05)$. Conversely, treatment with $\mathrm{gAd}$ increased the ATP level to $\sim 50 \%$ of that of the control group $(\mathrm{P}<0.05)$. However, compound $\mathrm{C}$ reversed the gAd-mediated increase $(\mathrm{P}<0.05$; Fig. 6$)$.

\section{Discussion}

The most prevalent cause of morbidity and mortality in patients with PQ poisoning is organ injury, in particular, injury to the 

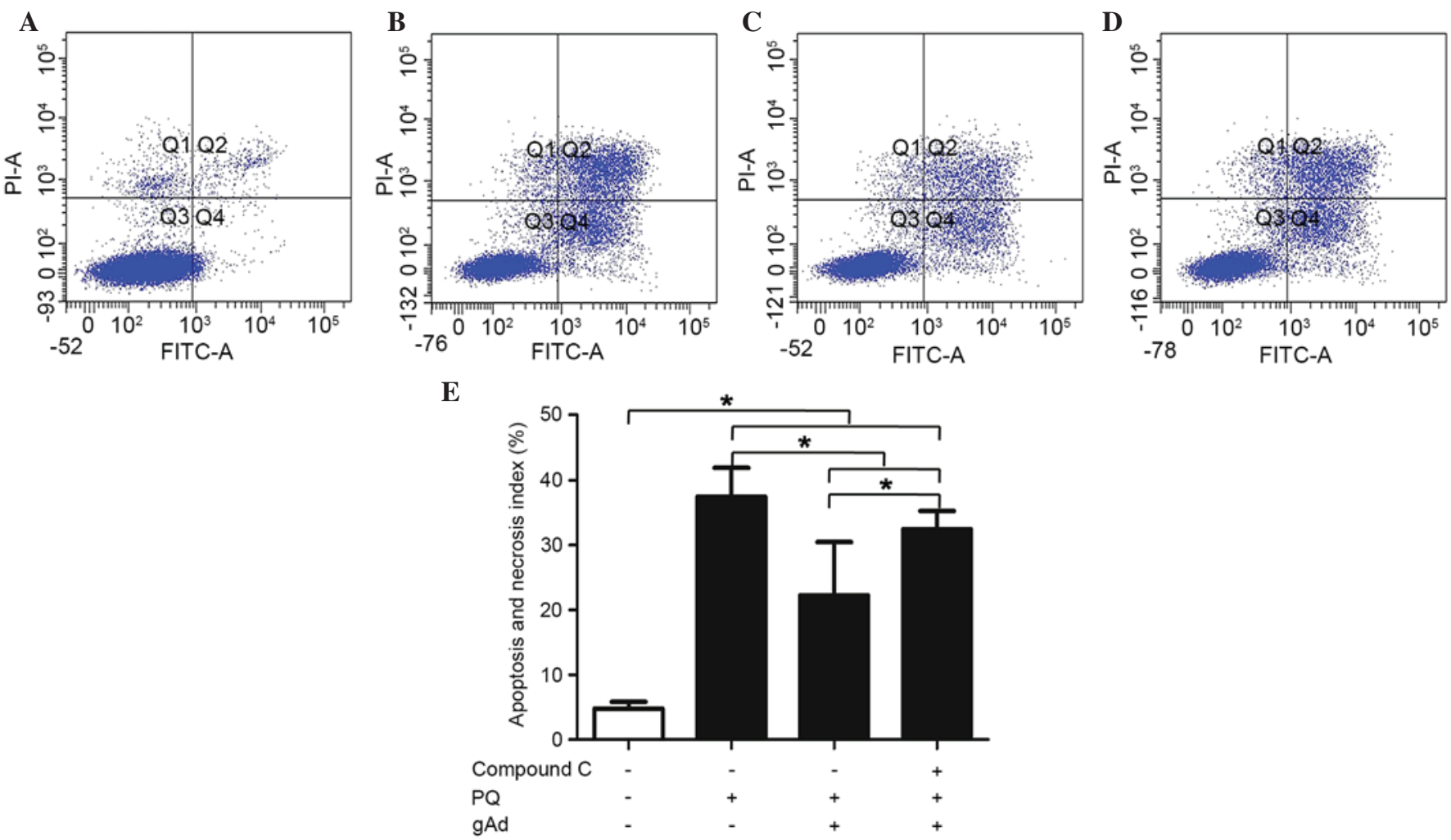

Figure 2. Anti-apoptotic effects of gAd on PQ-induced A549 cells. Cells were treated with $300 \mu \mathrm{M}$ PQ followed by $2.5 \mu \mathrm{g} / \mathrm{ml} \mathrm{gAd}$ with or without adenosine 5'-monophosphate-activated protein kinase inhibitor (compound C) pretreatment. The flow cytometry assay was conducted $24 \mathrm{~h}$ subsequent to administration of gAd. Flow cytometry analysis of the (A) control, (B) PQ, (C) PA and (D) PCA groups are presented. (E) Percentage of apoptosis and necrosis for the four groups are presented as the mean \pm standard deviation of five independent experiments, "P<0.05. gAd, globular adiponectin; PQ, paraquat; PI, propidium iodide; FITC, fluorescein isothiocyanate.
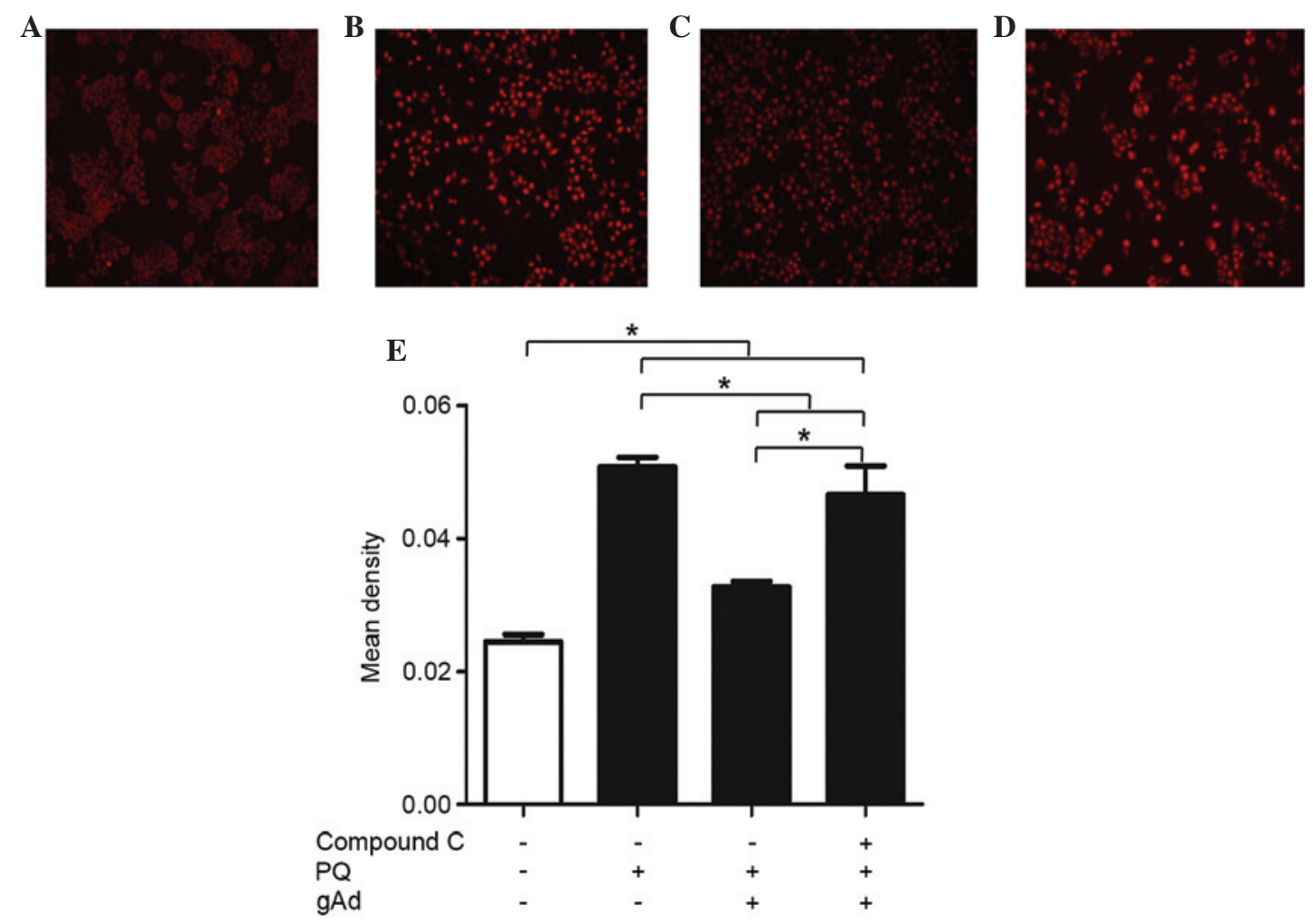

Figure 3. Anti-oxidative stress effects of gAd on PQ-induced A549 cells. Cells were treated with $300 \mu \mathrm{M}$ PQ followed by $2.5 \mu \mathrm{g} / \mathrm{ml} \mathrm{gAd}$ with or without adenosine 5'-monophosphate-activated protein kinase inhibitor (compound C) pretreatment. The fluorescence intensity of dihydroethidium was performed at $24 \mathrm{~h}$ after the administration of gAd. (A) Control group; (B) PQ group; (C) PA group; (D) PCA group; (E) Quantification of fluorescence data. The data are presented as the mean \pm standard deviation of five independent experiments, ${ }^{*} \mathrm{P}<0.05$. gAd, globular adiponectin; $\mathrm{PQ}$, paraquat. 


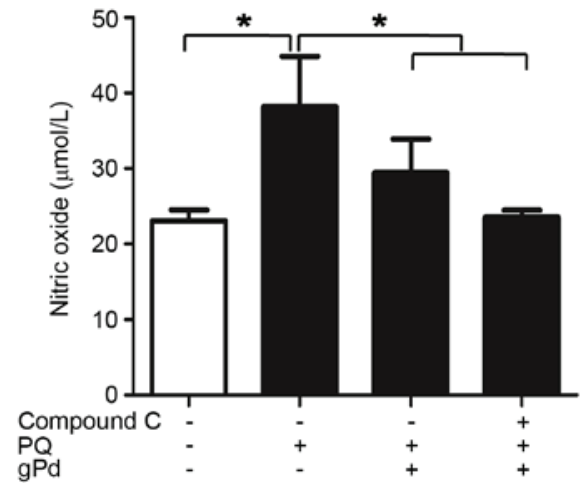

Figure 4. Anti-nitrative stress effects of gAd on PQ-induced A549 cells. Cells were treated with $300 \mu \mathrm{M}$ PQ followed by $2.5 \mu \mathrm{g} / \mathrm{ml} \mathrm{gAd}$ with or without adenosine 5'-monophosphate-activated protein kinase inhibitor (compound $\mathrm{C}$ ). A colorimetric assay was performed $24 \mathrm{~h}$ subsequent to the administration of gAd. The data are presented as the mean \pm standard deviation of five independent experiments, $\mathrm{P}<0.05$. gAd, globular adiponectin; $\mathrm{PQ}$, paraquat.
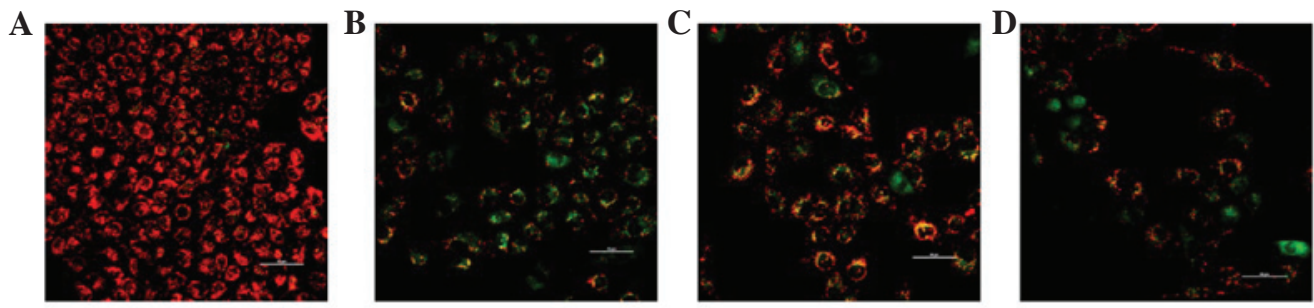

$\mathbf{E}$

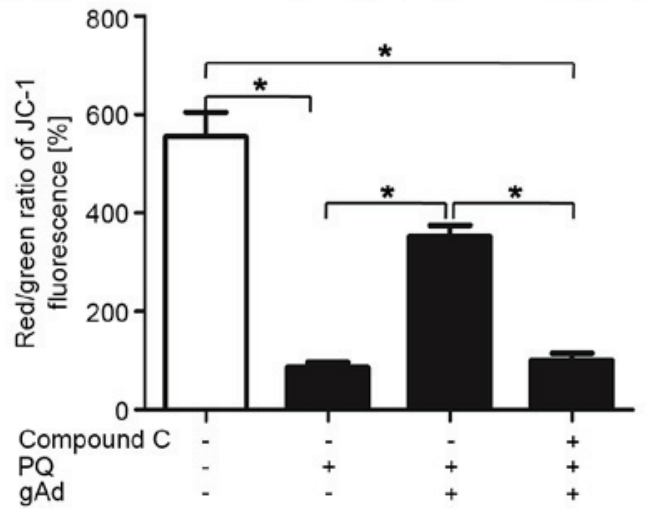

Figure 5. Effect of gAd on PQ-mediated $\Delta \Psi$ depolarization. Cells were treated with $300 \mu \mathrm{M}$ PQ followed by $2.5 \mu \mathrm{g} / \mathrm{ml} \mathrm{gAd}$ with or without adenosine 5'-monophosphate-activated protein kinase inhibitor (compound C). Confocal microscopy analysis was performed $24 \mathrm{~h}$ subsequent to the administration of gAd on the (A) control, (B) PQ group, (C) PA group and (D) PCA group. The red emission of the dye is due to a potential-dependent aggregation in the mitochondria, indicating high $\Delta \Psi$. Green fluorescence indicates the monomeric form of JC-1, appearing in the cytosol subsequent to mitochondrial membrane depolarization, indicating low $\Delta \Psi$. The ratio of red/green indicated the mitochondrial membrane depolarization states of different cells. (E) Quantification of the red/green ratio of the four groups is presented as the mean \pm standard deviation of five independent experiments, " $\mathrm{P}<0.05$. gAd, globular adiponectin; $\mathrm{PQ}$, paraquat; $\Delta \Psi$, mitochondrial membrane potential.

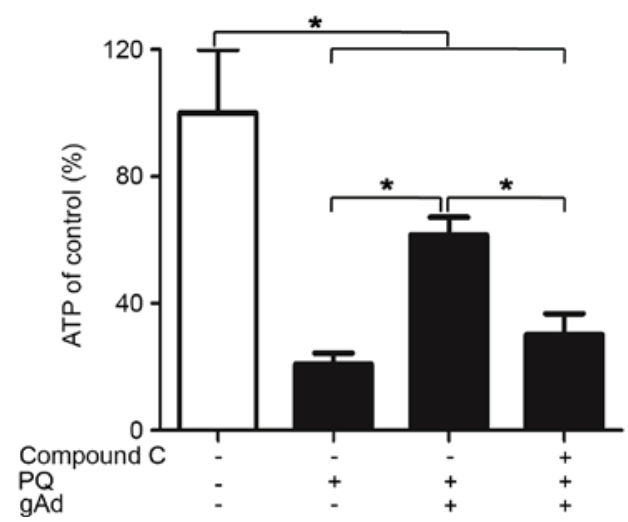

Figure 6. Effect of gAd on PQ-mediated reduction in ATP. Cells were treated with $300 \mu \mathrm{M}$ PQ followed by $2.5 \mu \mathrm{g} / \mathrm{ml} \mathrm{gAd}$ with or without adenosine 5'-monophosphate-activated protein kinase inhibitor (compound C). The luminometer assay was performed $24 \mathrm{~h}$ subsequent to gAd administration. The data are presented as the mean \pm standard deviation of five independent experiments, ${ }^{\prime} \mathrm{P}<0.05$. gAd, globular adiponectin; $\mathrm{PQ}$, paraquat; ATP, adenosine 5'-triphosphate. 
lungs (22). It has been demonstrated that excessive ROS/RNS stress resulting from impaired mitochondria, is an important feature of the injured lung epithelium in PQ poisoning, though the underlying mechanism responsible for the mitochondrial dysfunction remains unclear (23). Therefore, studies have focussed upon clarifying the underlying mechanisms for PQ-associated mitochondria injury of alveolar type II cells $(24,25)$ and developing novel strategies for the treatment of PQ-poisoned patients (26). In the present study, an established PQ poisoning model with A549 cells cultured with PQ for $24 \mathrm{~h}$ was used, and it was confirmed that apoptosis and cell death were exacerbated in the presence of PQ. Furthermore, it was indicated that PQ markedly disturbed mitochondrial function, depolarizing $\Delta \Psi$ and reducing ATP production, resulting in progressive increases of $\mathrm{O}_{2}^{-}$and $\mathrm{NO}$. This may be explained by the fact that $\mathrm{PQ}^{2+}$ induced the release of $\mathrm{Ca}^{2+}$ from cytoplasm (27), which may induce the depolarization of mitochondria and reduce $\Delta \Psi(28)$. This results in reductions of ATP synthesis to less than $50 \%$ of the normal level, thus activating pro-apoptotic and pro-necrotic proteins, including Bax and caspase 3/7/8/9 (29,30). As a result, the injured mitochondria produce excessive $\mathrm{O}_{2}^{-}$and $\mathrm{NO}$, which attached to the mitochondrial membrane in turn, and resulted in increases in ROS/RNS production and ultimately inducing apoptosis (31).

Injury resulting from $\mathrm{PQ}$ poisoning was however, reversed by treatment with gAd in the current study. Although the associated mechanisms remain to be fully elucidated, a previous study demonstrated the potential cellular protective effects of gAd against apoptosis via inhibiting mitochondrial dysfunction and ROS/RNS attachment (32). Therefore, the current study suggested that gAd may protect the alveolar type II cells aginst PQ-induced cell injury via inhibiting mitochondrial dysfunction and reducing oxidative/nitrative injury.

In order to further elucidate the underlying mechanisms for the cellular-protective effects of gAd in PQ poisoning against mitochondrial dysfunction and oxidative/nitrative injury, the signaling of AMPK, a protective kinase in oxidative/nitrative injury and a downstream mediator of gAd's mitochondrial protective actions, was investigated (33). It was identified that the protective effects of gAd could be reversed by an AMPK inhibitor, compound $\mathrm{C}$, which has been previously identified to block the cardiac protective benefits of $\mathrm{gAd}$ treatment against cardiac myocardial hypoxia/reoxygenation injury (15). This result clarified the critical role of AMPK in the pneumonocyte-protective effects of gAd, indicating it may be used in the therapy for PQ poisoning. This observation is consistent with a previous study by Yan et al (15), which demonstrated that $\mathrm{gAd}$ was able to activate AMPK-peroxisome proliferator-activated receptor $\gamma$ coactivator $1-\alpha$, resulting in increased ATP production, improving $\Delta \Psi$, thus leading to return to normal function. These results indicate that AMPK may be one of the key molecules in gAd's inhibition of mitochondrial dysfunction induced by PQ. However, interspecies differences $(34,35)$, animal model establishment $(36,37)$ and the injury procedure (38) may affect whether AMPK signaling serves a beneficial or detrimental role in the mitochondria. Experiments using animal models should be conducted in order to further clarify the mechanisms.

There were limitations in the present study. The inactivation of AMPK was not introduced when PQ was added to the cells, due to the high rate of mortality, thus the ability to directly demonstrate the role of AMPK in PQ-poisoned mitochondria following oxidative/nitrative injury was compromised. In addition, gAd treatment in PQ poisoning may modulate numerous other key molecules in addition to AMPK-ATP (39-41). Thus, investigation of these molecules in oxidative/nitrative injury requires additional investigation.

In conclusion, it was demonstrated that the $\Delta \Psi$ exhibited progressive reductions with increased oxidative/nitrative injury in PQ-induced apoptosis. In addition, it was identified that in PQ poisoning, $\Delta \Psi$ is increased in response to gAd treatment, which significantly attenuated mitochondrial dysfunction and oxidative/nitrative injury in PQ-poisoned alveolar type II cells. The present study provided indirect evidence for the possible role of the AMPK-ATP signaling pathway in gAd against oxidative/nitrative injury originating from the mitochondria following PQ poisoning. To the best of our knowledge, this is the first study to demonstrate the ability of gAd in attenuating oxidative/nitrative injury in PQ poisoning of lung cells beyond its metabolic actions. These observations add to the current understanding of the pathophysiology of PQ poisoning and the mechanisms of the widely used gAd therapy. This may aid in the development of future therapeutic strategies capable of assisting gAd in protecting against PQ-induced lung injury.

\section{Acknowledgements}

The current study was supported by the National Natural Science Foundation of China (grant nos. 30900493 and 81471836) and the Science and Technology Department Foundation of Sichuan Province (grant no. 2013JY0011). The authors would additionally like to acknowledge Professor Xin-liang Ma of Thomas Jefferson University (Philadelphia, PA, USA) for his help and advice aiding in the completion of the present study.

\section{References}

1. Vieira DN and de Azevedo-Bernarda R: Paraquat poisoning. Pulmonary lesions. J Toxicol Clin Exp 9: 177-186, 1989 (In French).

2. Cai Q, Lu Z, Hong G, Jiang X, Wu Z, Zheng J, Song Q and Chang Z: Recombinant adenovirus Ad-RUNrf2 reduces paraquat-induced A549 injury. Hum Exp Toxicol 31: 1102-1112, 2012.

3. Golbidi S, Botta A, Gottfred S, Nusrat A, Laher I and Ghosh S: Glutathione administration reduces mitochondrial damage and shifts cell death from necrosis to apoptosis in ageing diabetic mice hearts during exercise. Br J Pharmacol 171: 5345-5360, 2014.

4. Haagsman HP, Schuurmans EA, Batenburg JJ and Van Golde LM: Phospholipid synthesis in isolated alveolar type II cells exposed in vitro to paraquat and hyperoxia. Biochem J 245: 119-126, 1987.

5. Kurokawa H, Sugiyama S, Nozaki T, Sugamura K, Toyama K, Matsubara J, Fujisue K, Ohba K, Maeda H, Konishi M, et al: Telmisartan enhances mitochondrial activity and alters cellular functions in human coronary artery endothelial cells via AMP-activated protein kinase pathway. Atherosclerosis 239: 375-385, 2015

6. Barreto-Torres G, Hernandez JS, Jang S, Rodríguez-Muñoz AR, Torres-Ramos CA, Basnakian AG and Javadov S: The beneficial effects of AMP kinase activation against oxidative stress are associated with prevention of PPAR $\alpha$-cyclophilin D interaction in cardiomyocytes. Am J Physiol Heart Circ Physiol 308: H749-H758, 2015.

7. Berg AH, Combs TP and Scherer PE: ACRP30/adiponectin: An adipokine regulating glucose and lipid metabolism. Trends Endocrinol Metab 13: 84-89, 2002. 
8. Okamoto Y, Kihara S, Funahashi T, Matsuzawa Y and Libby P Adiponectin: A key adipocytokine in metabolic syndrome. Clin Sci (Lond) 110: 267-278, 2006.

9. Robinson K, Prins J and Venkatesh B: Clinical review: Adiponectin biology and its role in inflammation and critical illness. Crit Care 15: 221, 2011.

10. Kadowaki T, Yamauchi T and Kubota N: The physiological and pathophysiological role of adiponectin and adiponectin receptors in the peripheral tissues and CNS. FEBS Lett 582: 74-80, 2008.

11. Fang $X$ and Sweeney G: Mechanisms regulating energy metabolism by adiponectin in obesity and diabetes. Biochem Soc Trans 34: 798-801, 2006.

12. Tao L, Gao E, Jiao X, Yuan Y, Li S, Christopher TA, Lopez BL, Koch W, Chan L, Goldstein BJ and Ma XL: Adiponectin cardioprotection after myocardial ischemia/reperfusion involves the reduction of oxidative/nitrative stress. Circulation 115 : 1408-1416, 2007.

13. Kim JE, Song SE, Kim YW, Kim JY, Park SC, Park YK, Baek SH, Lee IK and Park SY: Adiponectin inhibits palmitate-induced apoptosis through suppression of reactive oxygen species in endothelial cells: Involvement of cAMP/protein kinase A and AMP-activated protein kinase. J Endocrinol 207: 35-44, 2010.

14. Wei CD, Li Y, Zheng HY, Sun KS, Tong YQ, Dai W, et al: Globular adiponectin protects $\mathrm{H} 9 \mathrm{c} 2$ cells from palmitate-induced apoptosis via Akt and ERK1/2 signaling pathways. Lipids Health Dis 11: 135, 2012

15. Yan W, Zhang H, Liu P, Wang H, Liu J, Gao C, Liu Y, Lian K, Yang L, Sun L, et al: Impaired mitochondrial biogenesis due to dysfunctional adiponectin-AMPK-PGC-1 $\alpha$ signaling contributing to increased vulnerability in diabetic heart. Basic Res Cardiol 108: 329, 2013.

16. Kim JE, Ahn MW, Baek SH, Lee IK, Ki YW, Kim JY, Dan JM and Park SY: AMPK activator, AICAR, inhibits palmitate-induced apoptosis in osteoblast. Bone 43: 394-404, 2008

17. Krysko DV, Vanden Berghe T, D'Herde K and Vandenabeele P. Apoptosis and necrosis: Detection, discrimination and phagocytosis. Methods 44: 205-221, 2008.

18. Radad K, Rausch WD and Gille G: Rotenone induces cell death in primary dopaminergic culture by increasing ROS production and inhibiting mitochondrial respiration. Neurochem Int 49: 379-386, 2006

19. Green LC, Wagner DA, Glogowski J, Skipper PL, Wishnok JS and Tannenbaum SR: Analysis of nitrate, nitrite and 15 N.nitrate in biological fluids. Anal Biochem 126: 131-138, 1982

20. Akao M, Ohler A, O'Rourke B and Marbán E: Mitochondrial ATP-sensitive potassium channels inhibit apoptosis induced by oxidative stress in cardiac cells. Circ Res 88: 1267-1275, 2001.

21. Yeh ST, Guo HR, Su YS, Lin HJ, Hou CC, Chen HM, Chang MC and Wang YJ: Protective effects of $\mathrm{N}$-acetylcysteine treatment post acute paraquat intoxication in rats and in human lung epithelial cells. Toxicology 223: 181-190, 2006.

22. Onyon LJ and Volans GN: The epidemiology and prevention of paraquat poisoning. Hum Toxicol 6: 19-29, 1987.

23. Gaudreault P, Karl PI and Friedman PA: Paraquat and putrescine uptake by lung slices of fetal and newborn rats. Drug Metab Dispos 12: 550-552, 1984.

24. Wang GY, Hirai K, Shimada H: Mitochondrial breakage induced by the herbicide paraquat in cultured human lung cell. J Electron Microsc (Tokyo) 3:181-184, 1992.

25. Chen YW, Yang YT, Hung DZ, Su CC, Chen KL:Paraquat induces lung alveolar epithelial cell apoptosis via Nrf-2-regulated mitochondrialdysfunction and ER stress Arch Toxicol 86:1547-58, 2012
26. Shokrzadeh M, Shaki F, Mohammadi E, Rezagholizadeh N, Ebrahimi F: Edaravone decreases paraquat toxicity in a549 cells and lung isolated mitochondria. Iran J Pharm Res 13: 675-681, 2014.

27. Chang X, Lu W, Dou T, Wang X, Lou D, Sun X, et al: Paraquat inhibits cell viability via enhanced oxidative stress and apoptosis in human neural progenitor cells. Chem Biol Interact 206: $248-255,2013$

28. Huang CL, Lee YC, Yang YC, Kuo TY and Huang NK: Minocycline prevents paraquat-induced cell death through attenuating endoplasmic reticulum stress and mitochondrial dysfunction. Toxicol Lett 209: 203-210, 2012.

29. Helewski KJ, Kowalczyk-Ziomek GI and Konecki J: Apoptosis and necrosis-two different ways leading to the same target. Wiad Lek 59: 679-684, 2006 (In Polish)

30. Kasahara A and Scorrano L: Mitochondria: From cell death executioners to regulators of cell differentiation. Trends Cell Biol 24: 761-770, 2014

31. Wang Z, Wang J, Xie R, Liu R and Lu Y: Mitochondria-derived reactive oxygen species play an important role in Doxorubicin-induced platelet apoptosis. Int J Mol Sci 16: 11087-11100, 2015.

32. Park M, Youn B, Zheng XL, Wu D, Xu A and Sweeney G: Globular adiponectin, acting via AdipoR1/APPL1, protects H9c2 cells from hypoxia/reoxygenation-induced apoptosis. PLoS One 6: e19143, 2011.

33. Lin Z, Wu F, Lin S, Pan X, Jin L, Lu T, Shi L, Wang Y, Xu A and Li X: Adiponectin protects against acetaminophen-induced mitochondrial dysfunction and acute liver injury by promoting autophagy in mice. J Hepatol 61: 825-831, 2014.

34. Zheng A, Li H, Xu J, Cao K, Li H, Pu W, Yang Z, Peng Y, Long J, Liu J and Feng Z: Hydroxytyrosol improves mitochondrial function and reduces oxidative stress in the brain of $\mathrm{db} / \mathrm{db}$ mice: Role of AMP-activated protein kinase activation. Br J Nutr 113 1667-1676, 2015.

35. Chen H, Wang JP, Santen RJ and Yue W: Adenosine monophosphate activated protein kinase (AMPK), a mediator of estradiol-induced apoptosis in long-term estrogen deprived breast cancer cells. Apoptosis 20: 821-830, 2015.

36. Chen LJ, Na R, Gu M and Ran QT: Reduction in mitochondria $\mathrm{H} 2 \mathrm{O} 2$ protects against insulin resistance through a mechanism of Akt and AMPK activation. Free Radic Biol Med 47: S85, 2009.

37. Pan JS, Huang L, Belousova T, Lu L, Yang Y, Reddel R, Chang A, Ju H, DiMattia G, Tong Q, et al: Stanniocalcin-1 inhibits renal ischemia/reperfusion injury via an AMP-activated protein kinase-dependent pathway. J Am Soc Nephrol 26: 364-378, 2015.

38. Wu SB, Wu YT, Wu TP and Wei YH: Role of AMPK-mediated adaptive responses in human cells with mitochondrial dysfunction to oxidative stress. Biochim Biophys Acta 1840: 1331-1344, 2014

39. Qiao LP, Kinney B, Yoo HS, Lee B, Schaack J and Shao JH: Adiponectin increases skeletal muscle mitochondrial biogenesis by suppressing mitogen-activated protein kinase phosphatase-1. Diabetes 61: 1463-1470, 2012

40. Zhou M, Xu A, Tam PK, Lam KS, Chan L, Hoo RL, Liu J, Chow KH and Wang Y: Mitochondrial dysfunction contributes to the increased vulnerabilities of adiponectin knockout mice to liver injury. Hepatology 48: 1087-1096, 2008.

41. Yamauchi T, Iwabu M, Okada-Iwabu M and Kadowaki T: Adiponectin receptors: A review of their structure, function and how they work. Best Pract Res Clin Endocrinol Metab 28: 15-23, 2014. 